\title{
Zero-energy edge states and chiral symmetry breaking at edges of graphite sheets
}

\author{
Shinsei Ryu ${ }^{\text {a,1 }}$, and Yasuhiro Hatsugai ${ }^{\text {a }}$

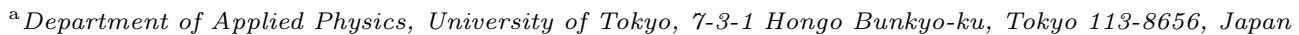

\begin{abstract}
Two-dimensional graphite sheets with a certain type of edges are known to support boundary states localized near the edges. Forming a flat band with a sharp peak in the density of states at the Fermi energy, they can trigger a magnetic instability or a distortion of the lattice in the presence of electron-electron or electron-phonon interactions. We shall discuss a relationship between chiral symmetry, which is the origin of the zero-energy edge states, and several types of induced orders such as spin density waves or lattice distortions. We also investigate electron correlation effects on the edge states for a wrapped quasi one-dimensional geometry, i.e., carbon nanotube, by means of the renormalization group and the open boundary bosonization.
\end{abstract}

Key words: carbon nanotube; edge states; chiral symmetry; chiral symmetry breaking;

\section{Introduction}

A fundamental aspect of two-dimensional (2D) graphite sheet is its intriguing band structure caused by the honeycomb network formed by $\sigma$-bonds $\left(s p^{2}\right)$ on which $\pi$ electrons are performing quantum mechanical hoppings. The nontriviality of the band can be quantitatively (and mathematically) characterized in terms of the Berry (Zak) phase, which is a phase degree of freedom in the quantum mechanical systems. The importance of the underlying gauge structure (the Berry phase) has also been recognized in the context of the integer quantum Hall effect or a theory of macroscopic polarizations[1].

A direct consequence of the nontriviality of the band is the appearance of edge states which are localized near the edges when the system is truncated with a certain type of edges. Indeed, Fujita et al. discovered peculiar edge states forming a flat band at zero-energy (the Fermi energy) in graphite sheets with zigzag or bearded (Klein) edges. [2]

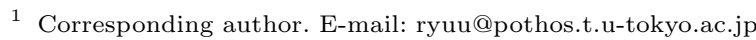

In the present paper, we consider edge states physics caused by electron correlation effects or coupling with lattice distortions on the honeycomb lattice with several types of edges. One of our main messages is that patters of order parameters [spin density waves (SDW), lattice distortions, etc.] which can be induced in the presence of interactions are dictated by a symmetry called chiral symmetry that is responsible for the existence of the zero-energy edge states (ZES's). Our argument is generic and can be applicable to other situations such as coexistence of time-reversal symmetry breaking order parameters at (110) surfaces of $d_{x^{2}-y^{2}-}$ wave superconductors. $[3,4]$

As an example, we shall discuss coupling between $\pi$ electrons and lattice distortions in terms of the $\mathrm{Su}-$ Schrieffer-Heeger (SSH) model. A possibility of a SDW order within the mean field theory (MFT) for the Hubbard model defined on the honeycomb lattice with edges is also considered. We also give detailed discussions on electron correlation effects when a graphite sheet are wrapped into quasi-1D geometry, i.e., carbon nanotube (CNT), where correlation effects are much more pronounced due to its low-dimensionality. The emergence of magnetic moments at edges is attractive 
since it opens up a possibility to have a new magnetic material made of exclusively light elements, without $d$ or $f$ - electrons. [5]

\section{Origin of zero-energy edge states and a Jahn-Teller-like argument}

Typical examples of a truncated graphite sheet that supports edge states are those terminated with zigzag edges in which edge states appear for $+2 \pi / 3<$ $\left|k_{y} a_{0}\right| \leq+\pi$, where $k_{y}$ is the wave number along the edges and $a_{0}$ the lattice constant (Fig. 1). On the other hand, a graphite sheet with armchair edges does not support edge states. The band structure for the zigzag case is nontrivial as its Zak phase is equal to $\pi$ for $k_{y}$ specified above. In the present case, nontriviality is also conveniently characterized in terms of a topological object, a set of loops in a parameter space, from which we can infer the existence of edges states. [3]

Although nontriviality of the band implies the existence of edge modes, it does not assure that edges states are located at zero-energy. Chiral symmetry, which means there is no matrix element connecting two sublattices in the present case, is another ingredient that dictates edge modes to be at zero-energy. Similar ZES's are also known for other systems such as surface

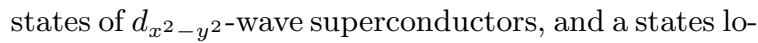
calized at a soliton in polyacetylene. All such examples can be understood in terms of the topology of bands and chiral symmetry in a unified fashion. [3]

As the edge states form a flat band (Fig. 1), giving rise to a sharp peak in the density of states (DOS) at the Fermi energy, a natural question is then what can happen in the presence of electron-electron or electronphonon interactions. Once we understand the origin of ZES's, it is possible to have some insights as to what kind of orders can occur as follows. Within the mean field picture, the band structure near the edges can be effectively modified when there are interactions to lift the degeneracy and hence lower the ground state energy. However, as ZES's are "protected" by chiral symmetry, such modifications should be accompanied with the breaking of chiral symmetry near the boundaries. This is in analogy with the Jahn-Teller theorem in which lattice distortions should break crystalline symmetries to lift degeneracy in electronic states. [4]

\section{Coupling with lattice distortions}

As a candidate of interactions that might lift the degeneracy of ZES's, let us consider coupling between the $\pi$ electrons and lattice distortions. We use the fol-

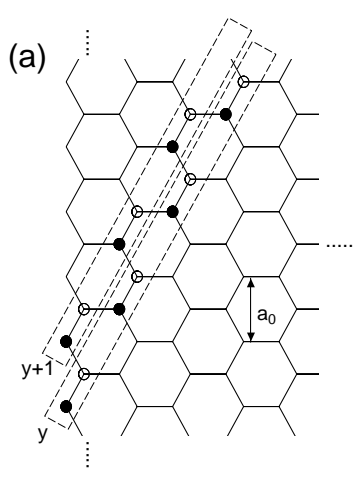

(b)

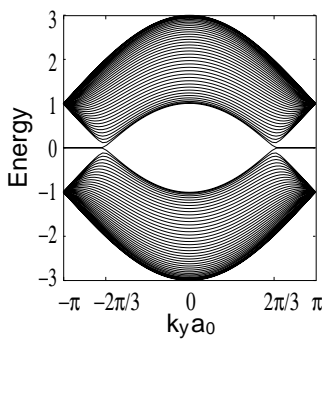

Fig. 1. (a) A $(N,-N)$ CNT with a zigzag edge. Unit cells are shown by broken lines. (b) The corresponding energy spectrum.

lowing simple model which is an extension of the SSH model to $2 \mathrm{D}$ graphite [6]:

$H_{\mathrm{SSH}}=\sum_{\langle i j\rangle \sigma}\left(-1+y_{i j}\right)\left(c_{i \sigma}^{\dagger} c_{j \sigma}+\right.$ h.c. $)+\frac{K}{2} \sum_{\langle i j\rangle} y_{i j}^{2}$

where $c_{i \sigma}^{\dagger} / c_{i \sigma}$ is an electron creation/annihilation operator at a site $i$ with spin $\sigma$. Here we treat $\sigma$-bonds as a classical spring with the tension $K$ and $y_{i j}$ represents the modulation of the length of a bond with a constraint $\sum_{\langle i j\rangle} y_{i j}=0$. We numerically determined $\left\{y_{i j}\right\}$ that minimizes the ground state energy, assuming the periodicity in $y$-direction with the unit cell shown in Fig. (1). Super structures with a larger unit cell can be energetically more favorable $[7,8]$, but the present choice is enough to demonstrate the relationship between chiral symmetry and ZES's.

When there are no boundaries and hence no edge modes, a first order transition with lattice distortions occurs at $K=K_{c} \sim 1.4$ as we decrease $K$. (Since the DOS is vanishing at the Fermi level, the transition occurs at a finite $K$.) When there are zigzag edges, one naively expects that the degeneracy between ZES's enhances Peierls instability and a lattice distortion near edges occurs at some critical value of $K=K_{c}^{\text {edge }}(<$ $K_{c}$ ). However, this is not possible as chiral symmetry is respected for any configuration of $\left\{y_{i j}\right\}$ in the above SSH model. Indeed, ZES's are stable with respect to lattice distortions as shown in Fig. (2).

For $K<K_{c}$, the bulk band structure is completely reconstructed by lattice distortions. The corresponding loops in a parameter space are trivial and there is no ZES even when one truncates the system by zigzag edges [Fig. (2)]. 


\section{Correlation effects and spin polarization}

\subsection{Mean field theory for $2 D$ geometry}

Another source of an instability, when there is a flat band formed by ZES's, is electron correlation, which can give rise to several kinds of orders, a typical example of which is the spin density wave. To model the correlation effects on edge states, let us consider the Hubbard model defined on the honeycomb lattice with zigzag edges [2]

$$
H=-\sum_{\langle i j\rangle \sigma}\left(c_{i \sigma}^{\dagger} c_{j \sigma}+\text { h.c. }\right)+U \sum_{i} n_{i \uparrow} n_{i \downarrow}-\mu \sum_{i \sigma} n_{i \sigma} .
$$

We adopt the following mean field ansatz

$$
\begin{aligned}
& H_{\mathrm{SDW}}=-\sum_{\langle i j\rangle \sigma}\left(c_{i \sigma}^{\dagger} c_{j \sigma}+\text { h.c. }\right)-\mu \sum_{i \sigma} n_{i \sigma} \\
& -U \sum_{i}\left\langle n_{i \uparrow}\right\rangle\left\langle n_{i \downarrow}\right\rangle+U \sum_{i}\left(\left\langle n_{i \uparrow}\right\rangle n_{i \downarrow}+\left\langle n_{i \downarrow}\right\rangle n_{i \uparrow}\right)
\end{aligned}
$$

to investigate possible magnetic structures, where $\left\langle n_{i \sigma}\right\rangle$ is determined self-consistently. If there is no boundary, the antiferromagnetic order occurs at $U=$ $U_{c} \neq 0$ (Again, the vanishing DOS at the Fermi energy allows the transition to occur at a finite $U$.). $U_{c}$ is estimated as 2.23 in MFT whereas $U_{c} \sim 4.5$ is obtained by the quantum Monte Carlo simulation [9].

In contrast to lattice distortions in the previous chapter, a finite $\left\langle n_{i \sigma}\right\rangle$ serves as an on-site potential to break chiral symmetry and hence lifts the degeneracy of ZES's. Indeed, Fujita et al.[2] found that magnetic moments are induced near boundaries even when $U<U_{c}$ within MFT. The calculated energy spectrum with the self-consistently determined mean field background $\left\langle n_{i \sigma}\right\rangle$ exhibits the splitting of branches of edge modes as shown in Fig. (2).

\subsection{Quasi $1 D$ geometry: carbon nanotube}

As we are discussing $2 \mathrm{D}$ systems with $1 \mathrm{D}$ edges, MFT is not reliable enough. Also, once we wrap a graphite sheet into quasi $1 \mathrm{D}$ tube geometry, i.e., CNT, the validity of MFT is much less obvious due to the quantum fluctuation enhanced by the low dimensionality. We are now to give detailed analyses for this geometry by means of the renormalization group (RG) and the open boundary bosonization. [10] Another quasi-1D geometry, which is a ribbon, was investigated by other authors. [11,12] Our main focuses are on (i) whether the localized charge and spin degree of freedom carried by ZES's can escape through the contact between the bulk conduction electrons and (ii) whether the total spin at
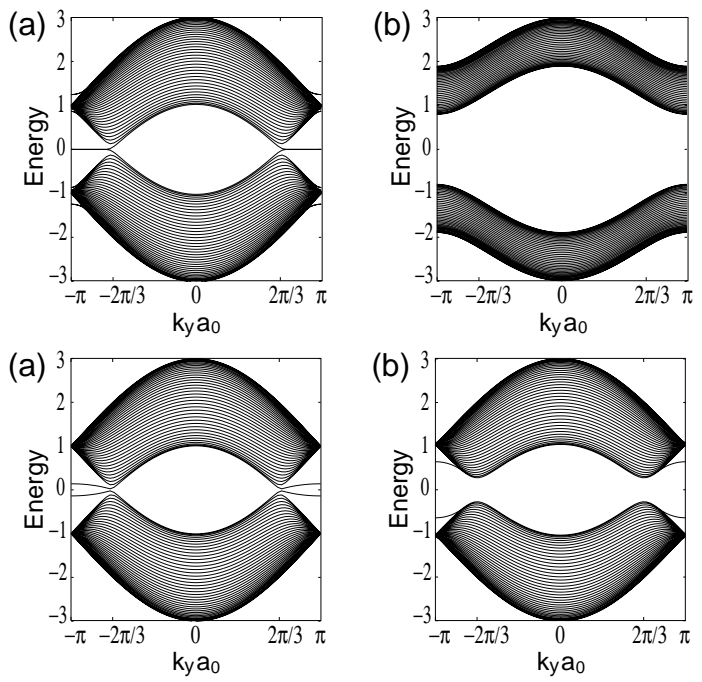

Fig. 2. Top: Band structures of $\pi$-electrons with lattice distortions for (a) $K=1.5>K_{c}$ and (b) $K=1.2<K_{c}$. Bottom: Band structures in the background of SDW mean field (a) $U=1.5<U_{c}$ and (b) $U=2.5<U_{c}$.

the boundary is polarized. The first question can be thought as a variant of the problem of quantum dissipation, examples of which include the Caldeira-Legget model, the X-ray edge problem, the Kondo effect, etc.

We consider a thin metallic $(N,-N)$ CNT, where there exist one $(N=6)$ or two $(N=9)$ ZES's when truncated with a zigzag edge. Starting from lattice models with the Coulomb or the Hubbard interaction, we can establish low-energy effective theories which describe correlation effects at boundaries. For the case of only one edge state, it is given by

$$
\begin{aligned}
H= & H_{0}+H_{I}, \\
H_{0}= & H_{c}+\epsilon_{e} \rho_{e}+U_{e} n_{\uparrow} n_{\downarrow}, \\
H_{I}= & \frac{v_{F} \lambda_{\rho}}{4} \rho_{e} \rho \\
& +v_{F} \lambda_{z} S^{z} J^{z}+\frac{v_{F} \lambda_{\perp}}{2}\left[S^{+} J^{-}+J^{+} S^{-}\right],
\end{aligned}
$$

where $H_{c}$ is the Hamiltonian for the conduction electrons that includes the forward scattering part of the Coulomb interaction by the open boundary bosonization (small back scatterings are neglected), $\boldsymbol{J}, \boldsymbol{S}$ represent the spin operator of the conduction electrons and ZES's, respectively, $\rho, \rho_{e}$ the density operator of the conduction electrons and ZES's, and $n_{\sigma}$ denotes the number operator for ZES's with spin $\sigma . v_{F}$ represents the Fermi velocity and an ultraviolet cutoff $\tau_{c}$ is introduced, which is of order of the inverse bandwidth. $U_{e}$ is the electron correlation between ZES's, $\epsilon_{e}$ the chemical potential for ZES's, and $\lambda_{\rho, z, \perp}$ represents a dimensionless coupling between the edge states and conduction electrons. Bare values of the Kondo-like couplings 
$\lambda_{z, \perp}$ (initial condition for RG analyses) are ferromagnetic due to the Fermi statistics; spin polarized states do not feel the Coulomb repulsion as the double occupancy is automatically prohibited due to the Pauli principle, and the kinetic energy is quenched for ZES's (Hund's law).

We perform a perturbative RG analysis up to oneloop order by infinitesimally rescaling the ultraviolet cutoff, $\tau_{c} \rightarrow \tau_{c} e^{-d l}$. From the resultant RG equations, it can be seen the bulk conduction electrons and edge states are completely decoupled in the infra-red $(l \rightarrow$ $+\infty), \lambda_{z, \perp} \rightarrow 0$. Furthermore, the repulsive bulk interactions are responsible to suppress the charge fluctuations at boundaries, and hence doubly occupying a edge state becomes unfavorable in the infra-red near half filling, $\varepsilon \rightarrow-\infty, U_{e}+2 \epsilon_{e} \rightarrow+\infty$. Then, we conclude that edge states do not diffuse into the bulk through the coupling with conduction electrons. Our RG analysis further indicates that a small deviation of $\epsilon_{e}$ from the half-filling $\left(\epsilon_{e}=0\right)$ is irrelevant due to renormalizations by $\lambda_{\rho}, \lambda_{z, \perp}$.

In order to see whether edge states tends to form a localized moments, we need to consider a thicker metallic CNT which supports more than two edge states. We then consider $(9,-9)$ CNT with zigzag edges for which edge states appear for $k_{y} a_{0}=-8 \pi / 9$ and $+8 \pi / 9$. The effective Hamiltonian for this case is given by

$$
\begin{aligned}
H_{0}= & H_{c}+\frac{I}{4} \rho_{1} \rho_{2}+K_{z} S_{1}^{z} S_{2}^{z}+\frac{K_{\perp}}{2}\left[S_{1}^{+} S_{2}^{-}+S_{2}^{+} S_{1}^{-}\right] \\
& +U_{e}\left[n_{1 \uparrow} n_{1 \downarrow}+n_{2 \uparrow} n_{2 \downarrow}\right]+\epsilon_{e} \rho_{e} \\
H_{I}= & \frac{v_{F} \lambda_{\rho}}{4} \rho_{e} \rho \\
& +v_{F} \lambda_{z} S^{z} J^{z}+\frac{v_{F} \lambda_{\perp}}{2}\left[S^{+} J^{-}+J^{+} S^{-}\right]
\end{aligned}
$$

where $\boldsymbol{S}_{\alpha}$ represents the spin operator for ZES's with $k_{y} a_{0}=+8 \pi / 9(\alpha=1)$ and $k_{y} a_{0}=-8 \pi / 9(\alpha=2)$, $\rho_{\alpha}$ the density operator, $n_{\sigma \alpha}$ the number operator, and $\boldsymbol{S}=\boldsymbol{S}_{1}+\boldsymbol{S}_{2}, \rho_{e}=\rho_{1}+\rho_{2}$. Initial conditions are given by $K_{z}=K_{\perp}=-I=-2 U_{e}<0, \epsilon_{e} \sim 0$, and $\lambda_{z}=\lambda_{\perp} \sim-\lambda_{\rho}<0$. RG equations for $\lambda_{z}, \lambda_{\perp}, h_{\epsilon}$ and $h_{U}$ are identical to those in the case of one edge state. Then, $\lambda_{z}$ and $\lambda_{\perp}$ become vanishing in the infrared limit, and charge fluctuations are suppressed $\varepsilon \rightarrow$ $-\infty, U_{e}+2 \epsilon_{e} \rightarrow+\infty$. On the other hand, RG equations for $K_{z, \perp}$ and $I$, which determine the total spin carried by the ground state of ZES's, are

$$
\begin{aligned}
& \frac{d h_{I}}{d l}=h_{I}-2 \lambda_{\rho}^{2}, \\
& \frac{d h_{K_{z}}}{d l}=h_{K_{z}}-\lambda_{z}^{2}, \quad \frac{d h_{K_{\perp}}}{d l}=h_{K_{\perp}}-\lambda_{\perp}^{2},
\end{aligned}
$$

where $h_{K_{z, \perp}}:=\tau_{c} K_{z, \perp}$ and $h_{I}:=\tau_{c} I$. We see that the Kondo couplings $\lambda_{z, \perp}$ renormalize the exchange interactions among edge states $K_{z, \perp}$, making it ferromag- netic. That is, the couplings between bulk and edge states turn out to assist the spin polarization, leading to the ground state with a highest spin $S=1$ at the boundary.

The result obtained here is consistent with spin polarization found in the mean-field theory in the previous section, a density matrix renormalization group study for a thin semi-conducting CNT [12], and an $a b$ initio local spin density functional calculation (LSDA) [13] for 2D sheet geometry.

\section{Conclusions}

ZES is a hallmark of the nontrivial band of a graphite sheet, and is related to rich physics. We have explored several types of order that can happen in the presence of electron-electron or electron-phonon interactions, with a main emphasis on chiral symmetry and its breaking. The Jahn-Teller-like argument presented in the present paper can be potentially applicable to other examples. As for spin polarization expected from chiral symmetry breaking, we investigated its possibility for thin metallic CNT's beyond the mean field treatment. Our RG analysis shows that bulk part of interactions enhance ferromagnetic interactions among edge states, leading to spin polarization at edges.

This work is supported by JSPS (S.R.) and KAWASAKI STEEL 21st Century Foundation.

\section{References}

[1] R.D. King Smith and D.H. Vanderbilt, Phys. Rev. B. 47, 1651 (1993); Phys. Rev. B. 48, 4442 (1993). R.Resta, Rev. Mod. Phys. 66, 899 (1994).

[2] M. Fujita, K. Wakabayashi, K. Nakada, and K. Kusakabe, J. Phys. Soc. Jpn. 65, 1920 (1996).

[3] S. Ryu and Y. Hatsugai, Phys. Rev. Lett. 89, 077002 (2002).

[4] S. Ryu and Y. Hatsugai, Physica C 388-389, 90 (2003).

[5] K. Kusakabe and M. Maruyama, Phys. Rev. B 67, 092406 (2003).

[6] M. Fujita, M. Igami and K. Nakada, J. Phys. Soc. Jpn. 66, 1864 (1997)

[7] K. Harigaya, Phys. Rev. B 45, 12071 (1992).

[8] K. Harigaya and M. Fujita, Phys. Rev. B 47, 16563 (1993)

[9] S. Sorella and E. Tosatti, Europhys. Lett. 19, 699 (1992).

[10] S. Ryu and Y. Hatsugai, Phys. Rev. B 67, 165410 (2003).

[11] Y. L. Lee, Y. W. Lee, Phys. Rev. B 66, 245402 (2002).

[12] T. Hikihara, X. Hu, H. H. Lin, and C. Y. Mou, cond-mat/0303159 
[13] S. Okada and A. Oshiyama, Phys. Rev. Lett. 87, 146803 (2001). 